Delft University of Technology

\title{
Observation of transition cascades in sheared liquid crystalline polymers
}

Fox, Ryan J.; Forest, M. Gregory; Picken, Stephen J.; Dingemans, Theo J.

DOI

10.1039/d0sm00275e

Publication date

2020

Document Version

Accepted author manuscript

Published in

Soft Matter

\section{Citation (APA)}

Fox, R. J., Forest, M. G., Picken, S. J., \& Dingemans, T. J. (2020). Observation of transition cascades in sheared liquid crystalline polymers. Soft Matter, 16(16), 3891-3901. https://doi.org/10.1039/d0sm00275e

\section{Important note}

To cite this publication, please use the final published version (if applicable).

Please check the document version above.

\section{Copyright}

Other than for strictly personal use, it is not permitted to download, forward or distribute the text or part of it, without the consent of the author(s) and/or copyright holder(s), unless the work is under an open content license such as Creative Commons.

\section{Takedown policy}

Please contact us and provide details if you believe this document breaches copyrights.

We will remove access to the work immediately and investigate your claim. 


\section{Observation of Transition Cascades in Sheared Liquid Crystalline Polymers}

Received 00th January 20xx, Accepted 00th January 20xx DOI: $10.1039 / \times 0 \times x 00000 x$

\author{
Ryan J. Fox ${ }^{a}$, M. Gregory Forest ${ }^{a, b}$, Stephen J. Picken ${ }^{c}$, and Theo J. Dingemans ${ }^{a, *}$
}

We report on the shear rheology of liquid crystalline solutions composed of charged, rodlike polymers that form supramolecular assemblies dispersed in water. Under steady shear, we observe shear thickening behavior, followed by a hesitation in the viscosity accompanied by an extremely narrow range of negative first normal stress difference. The Peclet number ( $P e$, shear rate normalized by rod rotational diffusivity) for the onset of shear thickening is in agreement with previous, high-resolution numerical simulations of the Doi-Edwards-Hess kinetic theory. We interrogate these dynamic responses through shear step-down experiments, revealing a complex evolution of transient responses. Detailed analysis of the stress transients provides compelling evidence that the principal axis of the rod orientational distribution, the nematic director, undergoes a cascade of transitions and coexistence of periodic states known as kayaking, tumbling, and wagging, before transitioning to steady flow alignment above a critical shear rate.

\section{Introduction}

Under steady shear, rodlike liquid crystalline polymers (LCPs) in the nematic phase notoriously exhibit either sustained oscillatory motion or steady alignment, both within and outside the shear plane (containing the flow $(x)$ and flow-gradient $(y)$ directions, orthogonal to the vorticity ( $z$ ) axis).1, 2 These shear responses give rise to remarkable rheological properties. In contrast, when an isotropic phase of LCPs is sheared, stationary alignment in the shear plane always occurs. ${ }^{3}$ In the nematic phase under shear, the temporal evolution of the principal axis of the rod orientational distribution function (ODF), termed the nematic director, either traces out closed orbits or sits at a stationary point on the 3D unit sphere. ${ }^{3,4}$ The periodic orbits of the director induce oscillations in the shear stress $\sigma_{x y}$ of a sheared nematic monodomain (a domain free of topological defects in the rod orientation field). Under transient flow of LCPs, such as flow reversal or start-up, the dynamic behavior of the rod ensemble can result in stress overshoots or undershoots, with significant implications for shear-dominated flow processing of these materials..$^{5-14}$

Qualitatively, the trajectories of the nematic director are used to classify the shear response of the entire ODF, described as residing within, oblique to, or perpendicular to the shear plane. When the director trajectory remains within the shear

a. Department of Applied Physical Sciences, University of North Carolina at Chapel Hill, Chapel Hill, NC 27599-3050, United States

b. Department of Mathematics, University of North Carolina at Chapel Hill, Chapel Hill, NC 27599-3250, United States.

c. Faculty of Applied Sciences, Delft University of Technology, Van der Maasweg 9 , 2629 HZ Delft, The Netherlands.

Electronic Supplementary Information (ESI) available: [details of any supplementary information available should be included here]. See DOI: 10.1039/x0xx00000x plane, the following states arise: tumbling (persistent rotation), wagging (finite oscillations about a mean angle $\alpha$ with respect to the flow direction), or flow-aligning (stationary at angle $\alpha$ with respect to the flow direction). ${ }^{3,4}, 11,15-18$ Simulations by Marrucci and Maffettone restricted to nematic orientation within the shear plane detailed each of these in-plane director states, where the wagging regime is the transition state between director tumbling and flow alignment.11, 16, 17 Later, unconstrained orientational simulations by Larson revealed new states where the director escapes the shear plane: kayaking (continuous rotation around the vorticity axis akin to the paddle of a kayaker) and logrolling (stationary alignment with the vorticity axis). ${ }^{19}$ Subsequent numerical simulations revealed yet new shear-induced states: tilted kayaking (rotation of the peak of the ODF about an axis between the vorticity axis and shear plane), out-of-plane steady (stationary alignment oblique to the shear plane), and chaotic states.3, 4, 20-25 Moreover, phase diagrams of all stable attractors ${ }^{3,4}$ reveal domains of coexistence of multiple stable states for a given strength $\varphi L / D$ of the nematic potential ( $\varphi$ is the rod volume fraction, $L / D$ is the rod aspect ratio) and imposed Peclet number $P e=\dot{\gamma} / D_{r}$, where $\dot{\gamma}$ is the shear rate and $D_{r}$ is the rod rotational diffusion coefficient. We note that, in general, the Doi-Hess-Edwards model simulations do not incorporate the effect of finite rod stiffness, which is known to enhance the tendency for the rods to adopt non-stationary director orbits. ${ }^{26-}$ 28 This effect from rod flexibility broadens the $P e$ range of unsteady, periodic director responses.

Historically, experimental results have been almost exclusively interpreted in the context of purely in-plane director states, i.e., tumbling, wagging, and flow alignment. However, it is now known that kayaking, rather than in-plane tumbling, is always selected as the preferred stable director state at low 
shear rates.3, 4 For example, Mewis et al. found an anomalous oscillatory regime in liquid crystalline solutions of poly- $\gamma$ (benzyl glutamate) (PBG) in $m$-cresol between the low-shear viscosity plateau and high-shear shear thinning. ${ }^{29}$ These authors concluded this was due to the transition from director tumbling at low shear rates, wagging at moderate shear rates, and finally in-plane flow-alignment at high shear rates. On a different model system, work by Lettinga et al. on nematic suspensions of the rodlike $f d$ virus invoked the tumbling-to-wagging transition to explain unusual shear thickening behavior. ${ }^{30,31}$ The results on nematic $f d$ virus suspensions are notable, in that they exhibit rheological behavior that indicates the elasticity associated with director heterogeneity, i.e., polydomain defect texture, is weak. As such, the imposed shear field strongly couples with the molecular ODF, similar to the situation in numerical simulations, and a broadening of the ODF due to director wagging caused the observed shear thickening behavior.

In addition, transient rheological experiments on liquid crystalline solutions of PBG in $m$-cresol by Grosso et al. found a narrow shear rate regime with sustained stress oscillations following step-down from high shear rates. ${ }^{22}$ Fourier transformation of the oscillating stress, in comparison to numerical simulations, led the authors to conclude that a combination of director wagging and logrolling must coexist over a certain shear rate regime, consistent with the most current numerical simulation results.3, 4, 20-25 However, the assignment of director wagging is uncertain because the associated strain period of stress oscillations from the kayaking and wagging states were shown to be identical by Tao et al. ${ }^{32-34}$ Director coexistence, suggested by Grosso et al., is only possible experimentally when sheared nematics exhibit a polydomain texture with orientational defects between monodomains. Furthermore, the initial-boundary conditions of nematic liquids influence the observed dynamic or stationary states at steadystate conditions. ${ }^{19}$ Moreover, specific surface anchoring conditions of the nematic director may lead to spatial heterogeneities in the director dynamics and local orientational order under shear. ${ }^{35,} 36$ We note that, even though logrolling is a stationary state with no director oscillations, when the shear rate $\dot{\gamma}$ of an initially flow-aligned sample is suddenly decreased into a lower $\dot{\gamma}$ regime, as in these authors' experiments, transient and long-lived oscillations manifest as a signature of the transition from flow-alignment to logrolling behavior. ${ }^{22}$ These results suggest that further experimental investigations must be informed by nematic director phase diagrams developed through high-resolution numerical simulations of the full ODF. 3,4

Herein, we report steady-state and transient rheological experiments on a nematic LCP that provide unprecedented signatures of transitions between, and coexistence of, dynamic director states. Moreover, these sheared nematic solutions exhibit an anomalous shear-thickening response that, in conjunction with previously reported numerical phase diagrams, are associated with dynamic transitions between attracting states of the ODF. The discovery of this shearthickening response in a polymeric nematic is notable, because shear thickening of a liquid crystalline phase has only been previously observed in nematic suspensions of rodlike $f d$ virus particles. ${ }^{30,}{ }^{31}$ Furthermore, our results lead us to conclude a different origin for the shear thickening regime, namely the kayaking state, rather than director wagging as suggested for nematic $f d$ virus. The results presented herein reveal that shear thickening, as predicted by numerical simulations of nematic monodomains, is experimentally observed if textural elasticity from the polydomain texture is weak.

\section{Materials and Methods}

2.1. Synthesis and Sample Preparation. The polymer was synthesized using a modified literature procedure previously reported. ${ }^{37-39}$ The reaction time of the interfacial polymerization was extended to $16 \mathrm{~h}$. After washing by four reprecipitations into acetone from water, the polymer was dried under vacuum. The intrinsic viscosity of the synthesized PBDT was measured in 96\% sulfuric acid using a suspended level Ubbelhode viscometer (CANNON Instrument Company, model E316). The Huggin's equation was fit to the specific viscosity divided by the mass concentration from a dilution series. The intrinsic viscosity was $2.58 \pm 0.02 \mathrm{dL} \mathrm{g}^{-1}$ and the Huggins coefficient was $0.52 \pm 0.02$. Polymer solutions were prepared by mixing PBDT and water and heating at $60{ }^{\circ} \mathrm{C}$ for 1 week. The samples were allowed equilibrate for over 1 month before measurements, and no aging effects were noted for the samples over the course of several weeks. Fully nematic phase behavior was observed at solution concentrations at $1.9 \mathrm{wt} . \%$ and above by polarized optical microscopy.

2.2. Rheology. For all rheological measurements presented, we employed a stress-controlled Anton Paar MCR-302 rheometer operating in strain-controlled mode with a $50 \mathrm{~mm} \boldsymbol{\beta}$ $=0.982^{\circ}$ cone and plate geometry. Preliminary experiments were conducted with a strain-controlled ARES-G2 rheometer with a dual-head design that decouples the strain application and torque measurements. The presence of steady-state shear thickening was confirmed using both rheometers. Moreover, the qualitative behavior of the stress transients in flow reversals and shear step-downs (i.e., non-strain scaling stress transients and large stress overshoots) were observed with both rheometers. We were only able to accurately measure the small normal forces under shear using the MCR-302, thus we only show the data collected with this rheometer. The temperature was controlled at $25 \pm 0.01^{\circ} \mathrm{C}$ by a circulating water bath under the bottom stationary plate. A low viscosity oil bath around the sample edges prevented evaporation that enabled sample stability over long time periods. Prior to any rheological measurements, the samples were pre-sheared for several thousand strain units at the highest shear rate investigated here $\left(1000 \mathrm{~s}^{-1}\right)$ until equilibration of the viscosity. This procedure ensured elimination of the sample's shear history due to sample loading. The calculated $N_{1}$ values were corrected for inertia by using $\boldsymbol{N}_{1}=\left[2 F_{z} /\left(\pi R^{2}\right)\right]+\left[\left(3 \pi \rho \omega^{2} \mathbf{R}^{4}\right) / 40\right]$, where $\omega=\boldsymbol{\beta} \dot{\gamma}$ 
is the angular rotation rate. ${ }^{40}$ The baseline in $\boldsymbol{N}_{\mathbf{1}}$ was determined by taking the average of the instrumental background for $100 \mathrm{~s}$ before and after measurement at each shear rate. During steady shear, $\boldsymbol{N}_{\mathbf{1}}$ was averaged over at least 500 strain units at steady state, after decay of the initial stress transient.

2.3. Small-angle X-Ray Scattering. SAXS measurements were taken of a 2.8 wt.\% PBDT solution in a thin-walled boronrich $1.5 \mathrm{~mm}$ diameter capillary (Charles Supper) with a SAXSLab Ganesha equipped with a Cu 50 kV Xenocs Genix ULD SL X-ray source producing an incident $X$-ray beam of $\lambda=1.54 \AA$ and a $2 D$ detector. The detector chamber is evacuated to $10^{-2} \mathrm{mbar}$ to reduce scattering from air. A sample-to-detector distance of $431 \mathrm{~mm}$ was used, and the $2 \mathrm{D}$ scattered intensity was azimuthally averaged over the scattering vector range of $0.05-$ $0.13 \AA^{-1}$ using the Nika package for Igor Pro $8 .{ }^{41}$

\section{Results and Discussion}

The LCP we investigate is a sulfonated all-aromatic polyamide (sulfo-aramid), poly(2,2'-benzidine-4,4'-disulfonyl terephthalamide) (PBDT), which self-assembles into helical rodlike assemblies (rods) with a diameter $D=0.8 \mathrm{~nm}$ when dissolved in water. ${ }^{37-39,}$, 22-46 We show the molecular structure and a schematic of the PBDT rods in Figure 1a. The high-aspectratio of PBDT rods is exemplified by the low isotropic-nematic $(\mathrm{I}-\mathrm{N})$ transition concentration, $c^{*}=1.2 \mathrm{wt} . \%$, and threshold for a fully nematic phase at $c^{* *}=1.9 \mathrm{wt} . \%$.

To estimate the PBDT rod dimensions, we employ the Onsager relation to the I-N concentration, given as $\varphi^{*}=4 / p$, where $\varphi^{*}$ is the critical volume fraction, and $p=L / D$ is the aspect ratio of the rigid rod. ${ }^{47,} 48$ This relationship provides an estimate of the aspect ratio of the constituent anisotropic rods that induce the formation of the nematic phase. Similarly, Picken and coworkers employed the Onsager relation to estimate of the dimensions of similar rodlike supramolecular assemblies of sulfonated derivatives of the well-known aramid, poly(para-phenylene terephthalamide) (PPTA). ${ }^{49,} 50$ Assuming a density of PBDT of $1.4 \mathrm{~g} \mathrm{~mL}^{-1}, \varphi^{*}=0.0085$ and $p=467$. The diameter of PBDT rods is $\sim 0.8 \mathrm{~nm}$; thus, the estimated $L$ is 373 $\mathrm{nm}$. This value for $L$ should be taken as an estimate of the contour length $\left(l_{c}\right)$ of the PBDT rods studied here, as higher molecular weight PBDT has been shown to result in a lower I-N transition concentration. ${ }^{51}$ Thus, the nematic PBDT rods studied here likely have $l_{c}$ smaller than their persistence length $\left(l_{p}\right)$, although exact values for $l_{p}$ of PBDT rods in aqueous solution have not yet been reported.

In Figure 1b, we show a polarized optical micrograph of a 2.8 wt.\% PBDT solution viewed within a $200 \mu \mathrm{m}$ pathlength rectangular glass capillary. The Schlieren texture confirms liquid crystallinity, and the unusually large domains on the order of $100 \mu \mathrm{m}$ suggests that the stresses arising from distortions of domain boundaries (i.e., Frank elasticity) are small in the quiescent state. ${ }^{52}$ Frank elastic stresses, which contribute to the
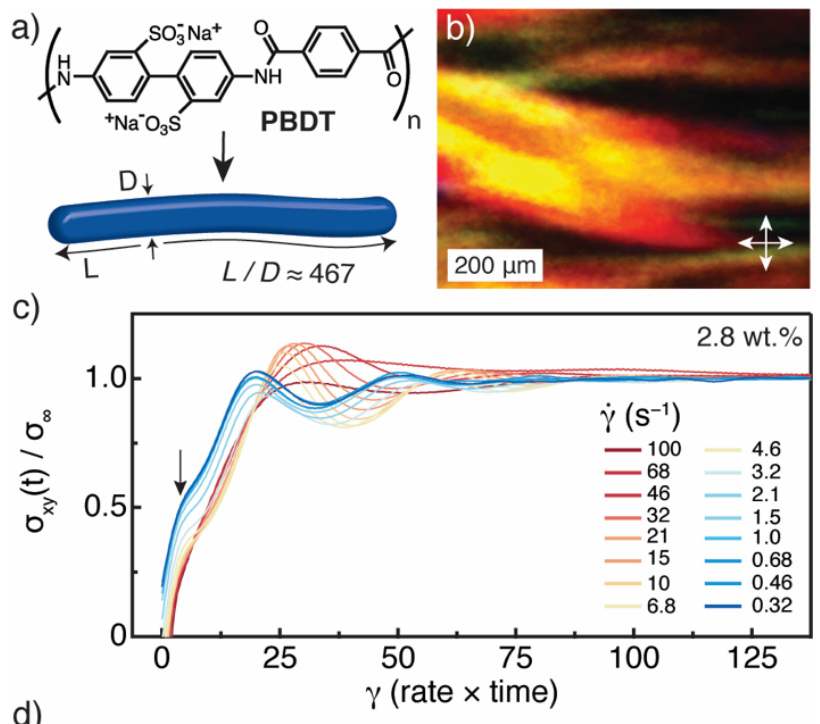

d)

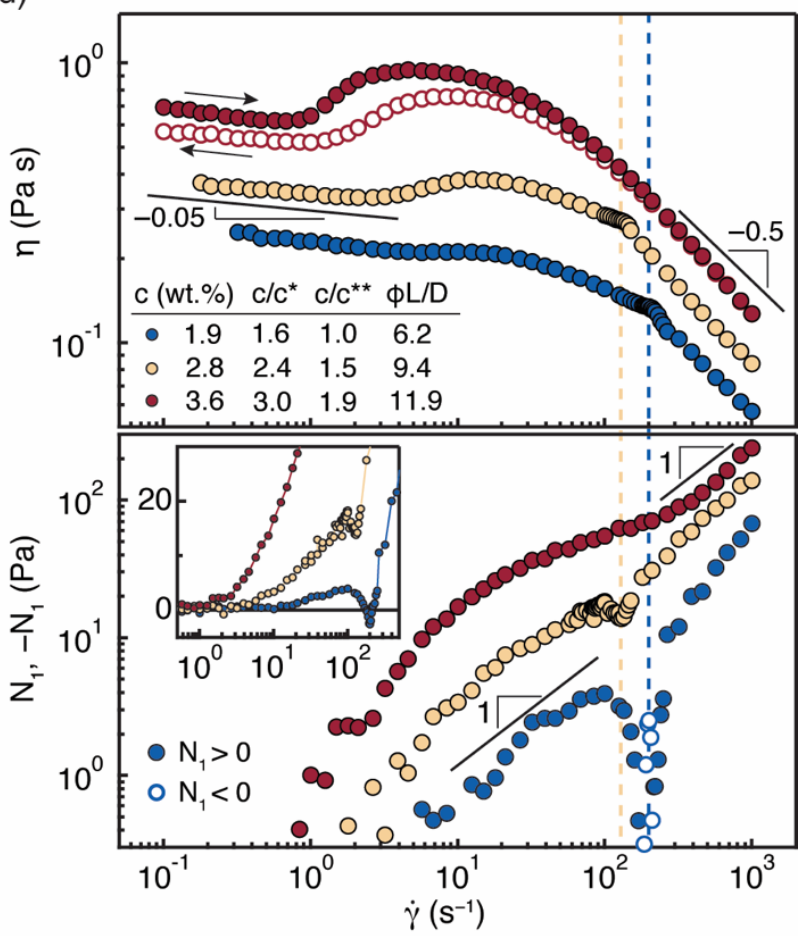

Figure 1. (a) Molecular structure and self-assembly of PBDT into rodlike assemblies. (b) Polarized optical micrograph of the polydomain texture of a $2.8 \mathrm{wt} . \%$ PBDT nematic solution in water. (c) Normalized shear stress $\sigma_{x y}(t) / \sigma_{\infty}$ following flow reversal of a $2.8 \mathrm{wt}$.\% solution at shear rates $\dot{\gamma}$ indicated in the figure legend. (d) Steady-state shear viscosity $\eta$ (top) and first normal stress difference $N_{1}$ (bottom) versus $\dot{\gamma}$ for nematic PBDT solutions. Open circles indicate decreasing $\dot{\gamma}$ (top) and negative $N_{1}$ values (bottom).

total stress of the flowing nematic, arise from gradients in the nematic director (i.e., the polydomain texture with disclinations/defects between monodomains). ${ }^{52,53}$ Stresses due to distortion of these disclinations under shear also contribute to the total stress measured.52, 54 Observations of sheared thermotropic nematics where a sudden proliferation of 
disclinations at a critical shear rate have been made, ${ }^{55}$ but shear thickening behavior directly associated with these defects has not been found. ${ }^{54}$ The length scales involved in these two contributions are mesoscopic in nature, on the order of $1-10$ $\mu \mathrm{m} .{ }^{56}$ Here, we observe that the domain features arising from disclinations are at least an order of magnitude greater in size than typically observed, similar to the observations of Lettinga et al. made on nematic $f d$ virus suspensions, ${ }^{30}$ and expect their contribution to the total stress will be correspondingly lower.

While we have not investigated the defect texture under shear of nematic PBDT solutions, our rheological measurements suggest that the stress contribution from director gradients are not dominant over experimentally accessible shear rates. First, we do not observe any evidence of so-called "Region I" shear thinning, ${ }^{57}$ which is characterized by a shear thinning exponent of the steady-state viscosity near -0.5 at low shear rates. ${ }^{58}$ In model LCPs, such as PBG, the nematic polydomain defect texture contributes significantly to the rheological response, even when Region I shear thinning is not observed. ${ }^{59-62}$ The stress contribution from the defect texture manifests itself by exhibiting strain-scaling behavior (i.e., collapse of the data when the transient normalized shear stress $\sigma_{x y}(t) / \sigma_{\infty}$ is plotted as a function of applied strain $\gamma=\dot{\gamma} t$ following flow reversal) under transient flow reversal experiments in the Newtonian regime of the flow curve. ${ }^{62}$ This strain scaling, generally expected for a solid-like response, is attributed to the viscoelastic response being dominated by the non-Brownian length scale associated with the nematic domain size. ${ }^{58,63-65}$

In Figure 1c, we show transient flow reversal measurements where the flow direction is suddenly reversed without changing the shear rate $\dot{\gamma}$ for $c=2.8 \mathrm{wt} . \%$. The transient $\sigma_{x y}(t) / \sigma_{\infty}$ versus $\gamma$ following flow reversal do not exhibit strain scaling behavior over any $\dot{\gamma}$ investigated. It is possible that strain scaling behavior may be recovered at lower shear rates, however the long times required for stress equilibrium and instrumental torque resolution precluded us from further investigation. Thus, the response of PBDT solutions under shear is dominated by coupling of the molecular orientational distribution function (ODF) to the imposed shear field, similar to the behavior observed in nematic $f d$ virus suspensions under shear. ${ }^{30,31}$

Further, we observe a shoulder in $\sigma_{x y}(t) / \sigma_{\infty}$ at small $\gamma$, labeled by the vertical arrow, that has been suggested to arise purely from the nematic fluid and distinct from the response of the nematic texture. ${ }^{61}$ It is interesting to observe that the shoulder in $\sigma_{x y}(t) / \sigma_{\infty}$ after reversal is strongly modulated as a function of $\dot{\gamma}$, even in the pseudo-Newtonian regime (for $c=$ $2.8 \mathrm{wt} . \%$, the pseudo-Newtonian regime spans the $\dot{\gamma}$ range of $0.32-3.2 \mathrm{~s}^{-1}$ ). In contrast, this shoulder remains constant within the strain scaling regime of highly textured nematics, which indicates that the molecular ODF is unperturbed by the shear flow. ${ }^{58,63-65}$ The strong dependence of this shoulder in flow reversals further supports our conclusion that the molecular ODF of the nematic PBDT rod ensemble couples to the shear field over the entire $\dot{\gamma}$ regime investigated here.
Through brief analysis of flow reversal experiments, we have established that the viscoelastic response of PBDT solutions is distinct from other polymeric LCPs in that the contribution of the polydomain nematic texture is low. Further differences arise when analyzing the steady-state flow curves of PBDT solutions under nonlinear, steady shear. We plot the steadystate viscosity $\eta$ (top) and first normal stress difference $N_{1}$ (bottom) of nematic PBDT solutions at $c=1.9,2.8$, and $3.6 \mathrm{wt} . \%$ in Figure 1d (top). The reduced concentrations $c / c^{*}$ and $c / c^{* *}$ and strength of the nematic potential $\varphi L / D$ are given in the figure legend. Note that all flow curves were constructed after a pre-shear (see Experimental section), and no significant hysteresis was observed upon increasing or decreasing $\dot{\gamma}$ for the two lower concentrations. However, for the $3.6 \mathrm{wt}$.\% solution, we plot flow curves with both increasing (filled circles) and decreasing (open circles) $\dot{\gamma}$, indicating a slight reduction in the magnitude of shear thickening response and low-shear rate viscosity after exposure of this sample to prolonged shearing at high shear rates.

Each sample exhibits three distinct regimes of $\eta$ versus $\dot{\gamma}$ behavior: a weak shear thinning regime at the lowest $\dot{\gamma}$ (power law slope $\approx-0.05$ ), followed by a continuous and reversible shear thickening, and finally shear-thinning at the highest $\dot{\gamma}$ used in the experiment (power law slope $\approx-0.5$ ). A hesitation in the flow curve precedes high- $\dot{\gamma}$ shear thinning for the two lower concentrations, indicated by the vertical dashed lines, while the transition is smooth at $c=3.6 \mathrm{wt} . \%$. This hesitation is a classic signature of the anomalous flow behavior of LCPs, and signifies the onset of flow-alignment, or "arrested tumbling" as termed by Larson, ${ }^{66}$ as observed in nematic PBG ${ }^{67}, 68$ and HPC $^{12}$ solutions under shear.

The unusual feature in the flow curves of nematic PBDT solutions is the existence of a pronounced shear thickening response in the moderate $\dot{\gamma}$ regime. The shear thickening response is qualitatively different than the behavior expected from the formation of a shear-induced network, as in dilute wormlike micelle solutions. In shear-thickening micellar solutions, the viscosity generally exhibits an induction period (prior to an increase in viscosity) ${ }^{69}$, accompanied by sustained temporal stress fluctuations under steady shear. ${ }^{69,} 70$ Moreover, shear-induced network formation is expected to result in a strong hysteresis effect, with increased viscosity and strong power law behavior as $\dot{\gamma}$ decreases towards zero. ${ }^{71,} 72$ In contrast, the lack of hysteresis in the $c=2.8 \mathrm{wt} . \%$ solution, which exhibits pronounced shear thickening, is not consistent with network formation. In addition, if network formation would occur at high $\dot{\gamma}$, we would expect that the final viscosity of the $c=3.6$ wt.\% sample would be higher than the initial viscosity, not lower (as we observe), when the shear rate is reduced from high to low rates during the hysteresis loop shown in Figure 1d (top).

We considered the influence of secondary flow instabilities or instrumental artifacts as a source of the observed shear 
thickening. First, we note that we observed shear thickening in the steady-state flow curves using two different rheometers (TA Instruments ARES-G2 and Anton Paar MCR-302, respectively). The geometries utilized for the two instruments have different surface treatments and configuration of the geometry of the sample edges. In the case of the ARES-G2, the lower plate (60 $\mathrm{mm}$ diameter, hardened chromium surface) extends beyond the diameter of the $50 \mathrm{~mm}$ cone (stainless steel surface), while in the case of the MCR-302 the lower fixture and upper geometry (both stainless steel surfaces) were exactly matched in terms of their diameter. It is unlikely that the exact same sample edge conditions between the two rheometers was realized; thus, edge effects were eliminated as an origin. Second, secondary flows from elastic instabilities are known to develop ${ }^{73}$ that can result in apparent shear thickening. However, the shear rates for which we observed shear thickening do not exhibit characteristics of this well-known flow instability, such as appearing after prolonged shearing. ${ }^{74}$ Finally, while we have not measured the velocity profile directly for the solutions reported herein, radial pressure measurements of sheared nematic PBG solutions are consistent with a viscometric flow profile, ${ }^{75}, 76$ and we did not observe any signatures of edge fracture. ${ }^{77}$ Thus, we turn to explain the observed shear thickening in terms of the dynamics of the nematic orientation distribution under shear.

If the observed shear thickening originates from a transition in the dynamics of the nematic director, we expect the onset to occur at similar $P e$ for all solutions, with variations that are predicted by numerical simulations of the rheological properties of these states. ${ }^{3,4}$ We calculated the $P e$ for the onset of shear thickening by scaling the onset $\dot{\gamma}$ to the rotational diffusion coefficient for a rod in a nematic, semidilute solution, given as $\bar{D}_{r} \cong 10^{3} D_{r, 0}\left(\bar{c} L^{3}\right)^{-2}\left(1-S_{e q}^{2}\right)^{-2}$, where $D_{r, 0}=$ $3 k_{B} T \ln (L / D) /\left(\pi \eta_{s} L^{3}\right)$ is the diffusion coefficient for a rod in dilute solution, $k_{B} T$ is the thermal energy, $\eta_{S}$ is the solvent viscosity, $\bar{c}$ is the rod number density, and $S_{e q}$ is the equilibrium scalar order parameter. ${ }^{78}$ While rigorous calculation of $\bar{D}_{r}$ requires explicit consideration of the orientational dependence, assuming it is constant does not qualitatively change the flowphase diagram in numerical simulations. ${ }^{4,19}$ For PBDT solutions deep into the nematic phase, as is the case here, $S_{e q}$ is high and does not vary with $c$ significantly; ${ }^{43}$ we use a value of $S_{e q}=0.886$ we measured by small-angle X-ray scattering (see Figure S1). This value of $S_{e q}$ is close to the saturation value observed in nematic $f d$ virus suspensions. ${ }^{79}$ The calculated $\bar{D}_{r}$ values are $1.95,0.84$, and $0.53 \mathrm{~s}^{-1}$ for $c=1.9,2.8$, and $3.6 \mathrm{wt} . \%$, respectively. In our measurements, the $\dot{\gamma}$ for the onset of shear thickening decreased by approximately one decade by increasing $c$ by a factor of $\sim 2$. However, we find that the onset of shear thickening occurs at an approximately constant $P e$ near 3 for each of the three solutions studied. This range of $P e$ has been correlated with bifurcations among stable director states by numerous numerical simulations.3, 4, 15, 19, 20, 23-25, 80 Despite the fact that there are a number of approximations in this calculation (i.e., the $L / D$ value is deduced from Onsager theory for rigid rods, neglect of electrostatic contributions on $\bar{D}_{r}$, use of $S_{e q}$ instead of experimentally measured order parameter under shear from the 1-3 and 1-2 shear planes, as done recently for $f d$ virus $^{81}$ ), we conclude that the origin of shear thickening lies with dynamical director state transitions that depend on a critical $P e$.

We gain further insight into the steady-state flow curve by the $N_{1}$ dependence on $\dot{\gamma}$ and how $N_{1}$ correlates with changes in $\eta$, plotted in Figure 1d (bottom). We took extreme care to control for the instrumental drift in the $N_{1}$ baseline during the steady-state measurements and ensured correct determination of the true zero level of $N_{1} .{ }^{82}$ Given that the absolute values of the normal force $F_{z}$ were small, we averaged over at least 500 strain units, and up to 2500 strain units for small values of $F_{z}$, after complete decay of the initial stress transient to improve resolution in $N_{1}$. Moreover, we applied standard corrections to the $N_{1}$ data to account for sample inertia, which are known to cause spurious negative $N_{1}$ regions. ${ }^{40,68}$ For all three solutions, $N_{1}$ scales linearly at low and high $\dot{\gamma}$, in accordance with prior experiments on other LCPs. ${ }^{67}, 83$ The lowest concentration, $c=$ 1.9 wt.\%, shows the most salient features between the three solutions studied here. After reaching a local maximum near $\dot{\gamma}=100, N_{1}$ then sharply decreases and becomes negative over a narrow range of $\dot{\gamma}$ and reaches its minimum value at $200 \mathrm{~s}^{-1}$, before sharply transitioning back to positive values. The inset of Figure 1d (bottom) shows a semi-log representation evidencing the positive-negative-positive transition in $N_{1}$ for $c=1.9 \mathrm{wt}$.\%.

The regime of negative $N_{1}$ is a hallmark feature in the shear rheology of LCPs, and arises from, in general, a broadening of the molecular ODF.15, 16 Tao et al. have shown through eventdriven Brownian dynamics simulations that broadening of the ODF due to director wagging originates from the loss of synchrony between the constituent rod motion and the director, thus decoupling the mesogens and director motion. ${ }^{32}$ This loss of synchrony causes a broadening of the molecular ODF, and results in a normal force that pulls the rheometer plates together. ${ }^{16}$ Note that, as we have discussed earlier, multiple director states may coexist over certain shear rate regimes, and this fact must be kept in mind in the following discussion. Given this fact, we conclude that the signatures of director wagging behavior are evident in nematic PBDT solutions at $\dot{\gamma}$ for which we observe negative $N_{1}$. Interestingly, we note that this regime is highly localized in $\dot{\gamma}$, in contrast to other LCPs that exhibit nearly one decade of $\dot{\gamma}$ with a negative $N_{1}$, depending on the solution concentration. ${ }^{12,67,68,84}$

With increasing concentration, the regime of negative $N_{1}$ is eliminated, but the $c=2.8 \mathrm{wt}$.\% retains a pronounced dip in $N_{1}$ at a slightly lower $\dot{\gamma}$ than the most negative value of $N_{1}$ at $c=$ $1.9 \mathrm{wt} . \%$. To the best of our knowledge, the observation of a dip in $N_{1}$, while remaining positive, has only been previously observed in highly concentrated solutions of HPC in m-cresol. ${ }^{77}$ Baek et al. suggested that either a change in director dynamics or an increase in the viscous stress contribution, in contrast to the elastic stress, would explain the elimination of a negative $N_{1}$ regime, while still exhibiting a dip in $N_{1}$. However, the enhanced 
contribution of the viscous stress contribution under flow could not explain the complete elimination of a local minimum in $N_{1}$, as observed in the melt phase of HPC77 and the PBDT solution at $c=3.6 \mathrm{wt}$.\% studied herein, and possibly reflects a change in the nature of the director dynamics. Here, we argue that director wagging with a negative contribution to the $N_{1}$ signal is likely present at $c=2.8 \mathrm{wt} . \%$, but in coexistence with a director state that contributes strongly to a positive $N_{1}$. At the higher solution concentration, we suggest that director wagging is completely suppressed, similar to the behavior of thermotropics that do not exhibit a regime of negative $N_{1} .{ }^{77,85}$, 86 It is remarkable to observe that a relatively low concentration PBDT solution, around $3 \mathrm{wt} . \%$, eliminates the director wagging behavior in a potentially similar manner to thermotropic LCPs.

Nematic $f d$ virus suspensions, a type of rodlike particle, also exhibit steady-state shear thickening, which Lettinga et al. attributed to the director wagging state. ${ }^{30,31}$ Moreover, they found strong stress oscillations in flow reversals with a minimum in strain period at the peak in the steady-state $\eta$. These authors suggested that the broadening of the molecular ODF from director wagging caused the increase in steady-state $\eta$. Unfortunately, the viscosity of $f d$ virus suspensions was too low to measure $N_{1}$, which would provide evidence for the transition from director tumbling to wagging in case of the presence of a negative $N_{1}$ regime. It is important to note that the shear thickening observed by these authors exhibited both qualitative and quantitative differences to the shear thickening shown here by PBDT solutions. First, the relative magnitude of $\eta$ change from shear thickening in $f d$ virus suspensions was smaller, around $10-15 \%$, compared to $\sim 50 \%$ observed for PBDT at $c=3.6 \mathrm{wt} . \%$. Second, the shear thickening observed in $f d$ virus occurred over a very narrow range of $\dot{\gamma}$, and immediately preceded high- $\dot{\gamma}$ shear thinning, whereas we observe a broad $\dot{\gamma}$ regime for shear thickening prior to the hesitation in $\eta$. Third, the onset $\dot{\gamma}$ for shear thickening in nematic $f d$ virus suspensions increased with increasing $c$, in contrast to decreasing $\dot{\gamma}$ with $c$ in PBDT solutions. These qualitative differences suggest that a different director state is responsible for the shear thickening observed in $f d$ virus suspensions in comparison to the shear thickening of PBDT solutions.

For nematic PBDT solutions, the signatures associated with the transition from coexistence of kayaking and tumbling to kayaking and wagging, i.e., the tumbling-to-wagging transition referred to by several authors, ${ }^{29,} 30$ are observed here between $\dot{\gamma} \approx 100-200$ for $c=1.9 \mathrm{wt} . \%$ and are well resolved from the shear thickening regime that occurs at lower $\dot{\gamma}$. Exhaustive numerical simulations by Forest et al. provide a guide for interpretation of the rheological behavior of nematic rodlike particles under shear, and we utilize their chosen terminology for the director states. ${ }^{3,4}$ Using these results in conjunction with our rheological measurements, we divide the steady-state flow curve for the $c=1.9 \mathrm{wt} . \%$ solution and assign the cascade of transitions in the nematic director response in
Figure 2. We caution that these assignments remain tentative, since small changes in concentration can dramatically change the series of stable directors possible for the nematic fluid under shear.

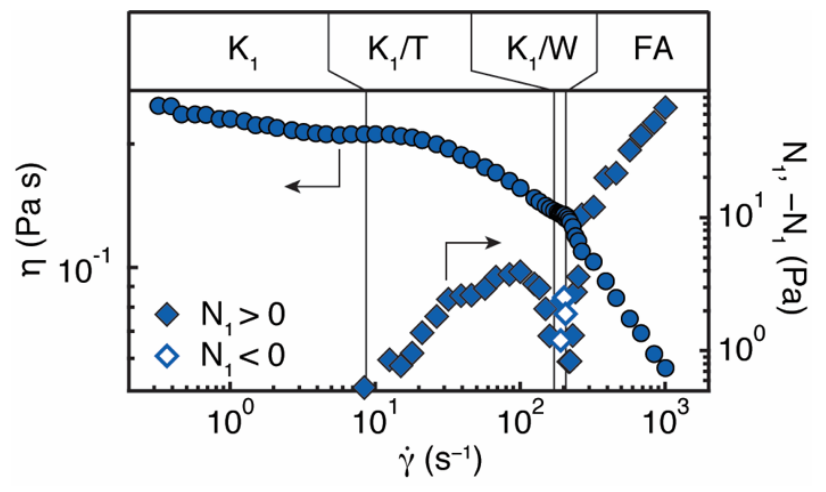

Figure 2. Steady-state flow curve of nematic PBDT in $\mathrm{H}_{2} \mathrm{O}$ at $c=$ 1.9 wt.\%, with the assignment of director state transitions as guided by the simulations of Forest et al.3, 4 The following director responses are suggested to occur as $\dot{\gamma}$ is increased: kayaking $\left(K_{1}\right)$, kayaking and tumbling $\left(K_{1} / T\right)$, kayaking and wagging $\left(\mathrm{K}_{1} / \mathrm{W}\right)$, and steady flow alignment $(\mathrm{FA})$.

At low $\dot{\gamma}$, kayaking is the unique stable state and exhibits an onset of shear thickening near $P e=3$, with increasing $N_{1}$ values, at a solution concentration slightly above the fully nematic concentration. The transition between bi-stable kayaking/tumbling, where kayaking and in-plane tumbling coexist, is predicted to occur at the transition between shear thickening and shear thinning in the steady-state viscosity. The transition from bi-stable kayaking and tumbling to kayaking and wagging occurs at the crossover from positive to negative $N_{1}$, occurring between $\dot{\gamma}=160$ and $180 \mathrm{~s}^{-1}$. At the maximum negative value of $N_{1}$, kayaking and wagging states yield to flow alignment. At $\dot{\gamma}$ above the onset of flow alignment, the viscosity monotonically decreases in a power law manner as the larger shear rate induces a narrower molecular ODF. We note that tilted kayaking and an out-of-plane steady state are predicted to be observed prior to flow alignment, but we cannot resolve rheological signatures that would provide evidence for them in these data.

Another director state that has been shown to be shear thickening is the logrolling state, where the average rod orientation is fixed along the vorticity axis. The logrolling attractor exhibits weak shear thickening and positive $N_{1}$ behavior, similar to kayaking which rotates about the vorticity axis. This director state is predicted for high enough concentrations and low $\dot{\gamma}$. Indeed, logrolling has been observed in a thermotropic polyester under shear; however, shear thickening was not observed. ${ }^{85,} 87$ Here, the magnitude of the viscosity increase we observe in nematic PBDT solutions at high concentrations with increasing $\dot{\gamma}$ is more consistent with the predictions for kayaking, rather than logrolling. 

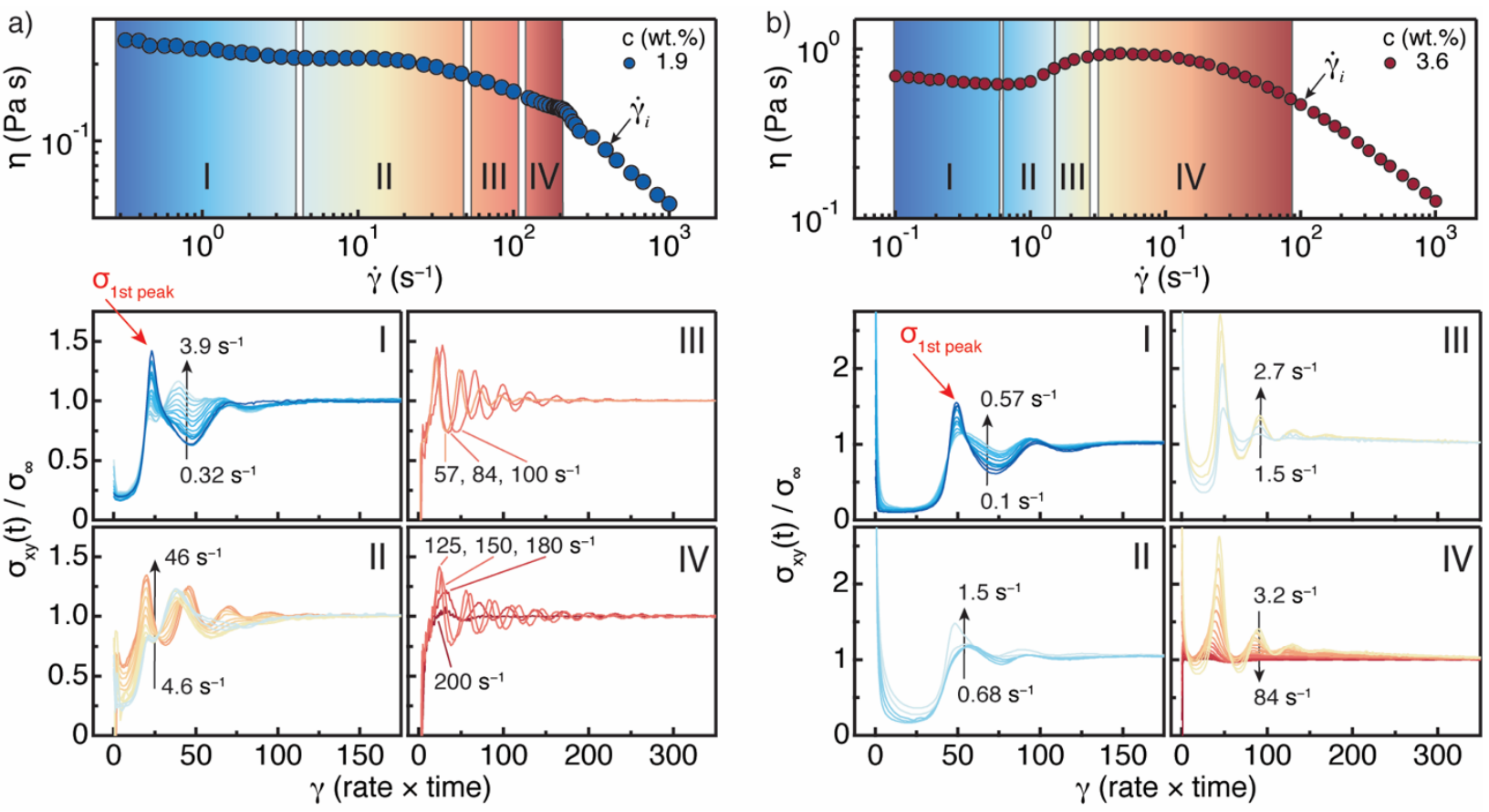

Figure 3. Steady-state flow curve (top) and $\sigma_{x y}(t) / \sigma_{\infty}$ as a function of $\gamma$ following shear step-down (bottom) at (a) $c=1.9$ wt.\% and (b) $3.6 \mathrm{wt} . \%$. The initial shear rate $\dot{\gamma}_{i}$ is indicated by the arrows on the flow curve. The final shear rates $\dot{\gamma}_{f}$ fall into zones labeled I, II, III, and IV. The zone color gradients labeled on the flow curve and colors of transient stress curves are correlated. Arrows in the plots of the transient stress indicate the direction of increasing $\dot{\gamma}_{f}$. The stress overshoot of the first peak is labeled by the red arrows (plotted as a function of the final shear rate in Figure $\mathbf{4 b}$ ).

To gain further insight into the unique rheological behavior of nematic PBDT solutions, we performed shear step-down transient experiments. First, we subject the sample to a high $\dot{\gamma}$ in order to prepare a steady flow-aligned ODF and then introduce a step change in $\dot{\gamma}$ without changing direction. This type of experimental protocol previously revealed an anomalous oscillatory regime associated with the wagging state in nematic PBG solutions in $m$-cresol. 22,29 We used an initial shear rate $\dot{\gamma}_{i}$ that was well within the flow-alignment regime, as determined by the local minimum in $N_{1}$ from the steady-state flow curves, to ensure a homogeneous and stationary director field. For $c=1.9$ and 3.6 wt.\%, we utilized $\dot{\gamma}_{i}=370$ and $100 \mathrm{~s}^{-1}$, respectively, as shown by the arrows in Figure $\mathbf{3 a}$ and Figure $\mathbf{3 b}$ (top). Exact calculation of the $P e$ that these $\dot{\gamma}_{i}$ correspond to is unreliable, as we have already shown that the molecular ODF is coupled to the shear field, thus $\bar{D}_{r}$ is a function of $\dot{\gamma}$ through its' dependence on $S$. After a steady state is reached at $\dot{\gamma}=\dot{\gamma}_{i}, \dot{\gamma}$ is reduced to a lower $\dot{\gamma}=\dot{\gamma}_{f}$ without changing the flow direction, and the transient stress is monitored as a function of total strain applied $\gamma=\dot{\gamma}_{f} t$. We have divided the flow curves into zones labeled I, II, III, and IV that correspond to the plots of the $\sigma_{x y}(t) / \sigma_{\infty}$ after step-down, in Figure $\mathbf{3 a}$ and Figure $\mathbf{3 b}$ (bottom). Note that these zones simply delineate the regions of $\dot{\gamma}_{f}$ used in the step-down experiments, and the color gradients correspond to the stress transient curve colors in the figures.

Through semi-quantitative analysis of the changes in the stress transient, we identify signatures of transitions in the director response from its' initial flow-aligned state. First, we discuss the transient behavior observed in Figure 3a (bottom) for $c=1.9 \mathrm{wt}$.\%. At low $\dot{\gamma}_{f}$, corresponding to the beginning of Zone I, the transient stress is characterized by an initial undershoot near $\sigma_{x y}(t) / \sigma_{\infty} \approx 0.2$, followed by a sharp overshoot that approaches 1.5 , then second local minima and maxima, before the oscillations are suppressed at higher $\gamma$. As $\dot{\gamma}_{f}$ is increased through Zone I, the qualitative behavior of the transient stress gradually evolves into a new form. The magnitude of the initial maximum decreases with $\dot{\gamma}_{f}$ while the peak $\gamma$ position remains constant. This reduction in the initial peak is concomitant with the emergence of a new, broader peak, with maxima near $\gamma=40$, that grows in intensity with increasing $\dot{\gamma}_{f}$ up to $3.9 \mathrm{~s}^{-1}$. This broader peak, which does not show any oscillatory behavior at larger $\gamma$, dominates the transient stress at the end of Zone I and beginning of Zone II. With increasing $\dot{\gamma}_{f}$ in Zone II, the opposite trend occurs as observed in Zone I. The broad peak (near $\gamma$ of 40 ) decreases in intensity and the sharp peak (near $\gamma$ of 20) increases, both maintaining constant $\gamma$ position. At a $\dot{\gamma}_{f}$ of $18 \mathrm{~s}^{-1}$, the broad peak (near $\gamma$ of 40) stops decreasing in intensity and begins to shift to higher $\gamma$. This apparent shift in peak position arises from the elimination of the prior broad peak and the emergence of a damped oscillatory signal that dominates towards the end of Zone II. This signal is distinct from the oscillatory behavior observed at the beginning of Zone I, where four peaks are now observed extending out to $\gamma$ of 70 and 95 at a shear rate of 46 $\mathrm{s}^{-1}$. 
Increasing the shear rate into Zone III, the oscillatory behavior becomes more pronounced and the damping is further inhibited. At a shear rate of 57 and $84 \mathrm{~s}^{-1}$, we observe up to 6 oscillations extending out to a strain of $\sim 140$. The peak positions and oscillation strain periods between these two $\dot{\gamma}$ are nearly identical. However, with a small increase in shear rate to $100 \mathrm{~s}^{-1}$, we observed a sudden shift of the first peak to higher strain and an increase in oscillation strain period, in addition to a further reduction in oscillation damping. The trend of increasing oscillation strain period and reduction in damping is continued into Zone IV, until at a shear rate of $200 \mathrm{~s}^{-1}$ the oscillatory stress transient is completely suppressed. We note that the suppression of oscillatory behavior at $200 \mathrm{~s}^{-1}$ exactly corresponds to the minimum value of $N_{1}$ in the steady-state flow curve.

The primary differences between the steady-state flow curve of the $c=3.6 \mathrm{wt}$.\% solution in comparison to $1.9 \mathrm{wt} . \%$ is the increased magnitude of shear thickening and elimination of the negative $N_{1}$ regime prior to flow alignment at high $\dot{\gamma}$. As a result, the zones labeled at 3.6 wt.\% are over different $\dot{\gamma}$ regimes than at $1.9 \mathrm{wt} . \%$. However, the qualitative change in the stress transients at $3.6 \mathrm{wt}$.\% with $\dot{\gamma}$ are similar to $1.9 \mathrm{wt} . \%$, but the oscillatory behavior is broader and more damped. The stress undershoots in Zone I and II reach lower values and remain in a low-stress state for larger strain values than at 1.9 wt.\%. That is, higher $c$ enables the flow-aligned state to be maintained for larger strain values at low $\dot{\gamma}$ following stepdown.

As we have previously discussed, director wagging is associated with the hesitation in the flow curve and negative $N_{1}$, observed at $c=1.9 \mathrm{wt} . \%$. At $3.6 \mathrm{wt} . \%$, this negative $N_{1}$ regime, and the corresponding strongly oscillating stress transients (see Zone IV for $c=1.9 \mathrm{wt} . \%$ ), are eliminated. Moreover, the transition from shear thickening to shear thinning at high $\dot{\gamma}$ for $c=3.6$ wt.\% in the steady-state measurement is reflected in a more gradual elimination of oscillatory behavior in Zone IV with increasing $\dot{\gamma}$. Thus, we conclude the stress transient signature associated with director wagging is present at $c=1.9 \mathrm{wt} . \%$, but is suppressed at 2.8 and entirely eliminated at $3.6 \mathrm{wt} . \%$.

We further analyze the shear step-down experiments by plotting $\eta$ normalized to its value at $0.5 \mathrm{~s}^{-1}$, and the normalized stress overshoot value of the first peak, $\sigma_{1 \text { st peak }} / \sigma_{\infty}$, following step-down as a function of $\dot{\gamma}$ in

Figure $4 \mathbf{a}-\mathbf{b}$, respectively. The shear rate dependence of $\sigma_{1 \text { st peak }} / \sigma_{\infty}$ reports on the nature of the transient behavior of the nematic director as it evolves from the initial, stationary, flow-aligned state (at high shear rates) to the final, periodic, state at lower shear rates (i.e., kayaking, tumbling, wagging, or coexistence of states). The strong first peak following stepdown, labeled with red arrows in Figure 3, observed at the beginning of Zone I, systematically decreases in its' normalized stress value as the shear rate increases into the beginning of Zone II (i.e., onset of shear thickening). At the onset of steady- state shear thickening, we find that $\sigma_{1 \text { st peak }} / \sigma_{\infty}$ then rapidly increases in value with increasing shear rate, concomitant with the emergence of long-lived stress oscillations. Thus, we conclude that the shear rate dependence of $\sigma_{1 \text { st peak }} / \sigma_{\infty}$ and steady-state viscosity are essentially signatures of the same underlying director response.

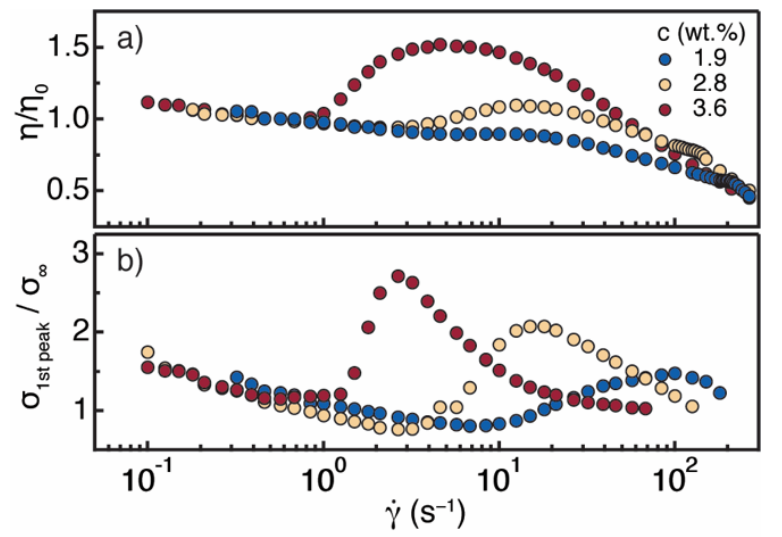

Figure 4. (a) Normalized steady-state flow curve and (b) normalized stress overshoot value of the first peak following shear step-down as a function of shear rate (see red arrows in Figure 3). The initial shear rates in (b) are 370, 230, and 100 for $c=1.9,2.8$, and $3.6 \mathrm{wt} . \%$, respectively.

At low $\dot{\gamma}$, the normalized $\eta$ collapse onto each other and follow similarly weak shear thinning behavior with increasing $\dot{\gamma}$ up to $\sim 1 \mathrm{~s}^{-1}$, consistent with similar director dynamics between the three solutions at low $\dot{\gamma}$. As shown previously, the onset of shear thickening occurs at different $\dot{\gamma}$, but similar $P e$. The dependence of the stress overshoot on $\dot{\gamma}$ shows that the overshoot values also collapse at low $\dot{\gamma}$ and decrease with increasing $\dot{\gamma}$ up until the onset of shear thickening. For $c=1.9$ and $2.8 \mathrm{wt} . \%$, the stress overshoot dips below unity prior to increasing, while at $3.6 \mathrm{wt} . \%$ the stress overshoot is always greater than unity. The shear thickening observed in the flow curve correlates with an increase in the stress overshoot of the first peak. The sharpness of the transition from decreasing to increasing stress overshoot value apparently correlates with the relative magnitude of the steady-state shear thickening response.

To understand this unusual behavior in the stress overshoot, we compare to the results of Mewis et al. on nematic PBG solutions. ${ }^{29}$ These authors reported an anomalous oscillatory regime following shear step-down that they attributed to director wagging, in contrast to director tumbling behavior they suggested within the strain-scaling region of the flow curve. The transient stress data following step-downs within a narrow range of $\dot{\gamma}$ exhibited strong oscillations that were damped after $\gamma$ values greater than 100 . Normalization of the transient stresses show the stress overshoot values of the first peaks were very close to unity. Similarly, the flow reversal results of Lettinga et al. on nematic $f d$ virus suspensions in the shear rate 
regime they assign to director wagging show stress overshoot values that reach, at most, normalized stress values of near 1.1. ${ }^{30}$ That is, the stress overshoots observed in PBDT solutions that approach normalized stress values in excess of 2.5 (at 3.6 wt.\%) following step-downs is inconsistent with the behavior generally associated with the director wagging regime in the literature. As such, we suggest that the large stress overshoots are signatures for the occurrence of the kayaking director state as it evolves from a flow-aligned condition to its' out-of-plane periodic orbit, centered along the vorticity axis. Presumably, the change in director orientation from an in-plane stationary state (flow alignment) to the out-of-plane orbit in kayaking results in a strong elastic response that manifests as significant stress overshoots in the transient rheological experiments.

Extracting the strain period of the oscillations, which is directly related to the rotational period of the director, is not straightforward as our step-down measurements exhibit complex behavior and evolution with $\dot{\gamma}$. Moreover, manual measurement of the stress oscillations (i.e., strain period between stress peaks) will yield a single oscillation period, when there may be two or more frequency components present. ${ }^{22}$ Thus, we decomposed the transient stress into its component frequencies by utilizing a fast Fourier transform (FFT) of the transient oscillatory stress. We show the FFT spectra for $c=1.9$ wt.\% in Figure 5 over a wide range of $\dot{\gamma}_{f}$. As we integrate over $\gamma$ in the FFT, the ordinate of the power spectra is the inverse strain period, $P=1 / \gamma=T \dot{\gamma}$, where $T$ is the oscillation time. In addition, we plot the predictions of the strain period for the kayaking/wagging states of Tao et al. found by Brownian dynamics simulations, ${ }^{32-34}$ which gives the oscillation period as $P=4.2 \varphi L / D$. Note that they found $P$ to be nearly constant in both the kayaking and wagging states. This prediction systematically overpredicts $P$ for nematic $f d$ virus suspensions, ${ }^{30}$ but this discrepancy was attributed to rod flexibility. ${ }^{28}$

The peaks observed in the power spectra relate to the constituent frequency components of the transient oscillatory stress following step-down. Close to the origin, more strongly observed at low $\dot{\gamma}$, we observe a signal that matches the strain period of a full rotation of the motor corresponding to $\gamma \cong$ $\phi / \beta=2 \pi / 0.017139=366.6, \quad 1 / \gamma=1 / 366.6=0.0027$, where $\phi$ is the motor angular deflection, and hence ignored. The FFT spectra peaks are broad, due to the damped nature of the oscillating transient stresses. ${ }^{88}$ In the power spectra at low $\dot{\gamma}$, we clearly observe the presence of multiple frequency components within the transient stress, as previously suggested by visual inspection of Figure 3a (bottom). At low $\dot{\gamma}$, a double topped feature exists, which gives way to a single strong band near $1 / \gamma=0.018$. Further increases in the $\dot{\gamma}$ into the negative $N_{1}$ regime cause this band to be suppressed and a narrower band near $1 / \gamma=0.038$ to grow in. This band, which dominates the FFT spectra over the $\dot{\gamma}$ regime that we observe a negative $N_{1}$ for this $c$, is in quantitative agreement with the predictions for the oscillation period found by Tao et al. for kayaking/wagging in their simulations, shown as the dashed vertical line. ${ }^{32-34}$

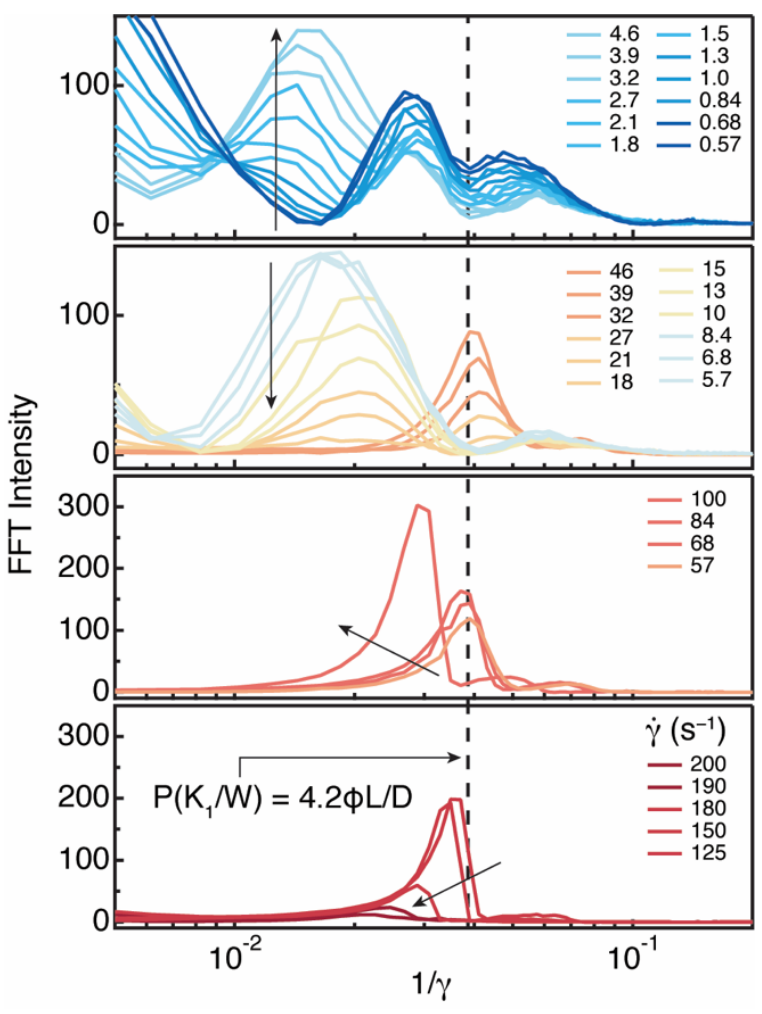

Figure 5. Fourier transform power spectra of transient stress following shear step-downs at $c=1.9 \mathrm{wt} . \%$. The initial shear rate was $370 \mathrm{~s}^{-1}$ and the final shear rates are given in the figure legends. Solid arrows indicate increasing $\dot{\gamma}_{f}$, and the vertical dashed line is the tumbling period prediction by Tao et al. for both the kayaking and wagging director states. ${ }^{32-34}$

With further increases in $\dot{\gamma}$ up to $200 \mathrm{~s}^{-1}$, as the transition to flow alignment occurs, the FFT peak shifts to lower $1 / \gamma$ (higher $\gamma$ period), and reduces in intensity. This signifies the suppression of the oscillatory behavior of the nematic director by increasing its strain period and decreasing its intensity as it progresses to stationary flow aligning behavior following stepdown. Our data suggests that a constant $P$ exists only over a limited $\dot{\gamma}$ regime, and more complex behavior of the nematic director that encompasses director state coexistence must be considered to fully explain the flow behavior that we observe in liquid crystalline solutions of PBDT rods. Direct measurement of the director orientation under shear using time-resolved X-ray scattering under the transient flow conditions utilized in this work would provide more conclusive understanding of the rheological behavior we observe in nematic PBDT solutions. ${ }^{89,} 90$

\section{Conclusions}

In conclusion, we have reported on the steady-state and transient rheology of a nematic solution of charged, rodlike polymer aggregates that exhibit anomalous shear thickening 
and negative $N_{1}$ behavior. We identify the origin of shear thickening to "director kayaking", as predicted by prior numerical simulations of the coupled kinetic-hydrodynamic theory for nematic rodlike polymers.3,4 Our results, in conjunction with the results of Lettinga et al. on nematic $f d$ virus suspensions, ${ }^{30}, 31$ provide experimental evidence that shear thickening in nematic solutions may originate from at least two distinct responses, described in terms of the principal axis, or nematic director, of the orientational distribution of the rod ensemble. We revealed the coexistence of unsteady, periodic director states by Fourier analysis of the transient stress following shear step-down, as found over a narrow range of shear rates for PBG solutions. ${ }^{22}$ Over a limited $\dot{\gamma}$ regime, we found agreement of the experimental oscillation periods we assigned as kayaking/tumbling and kayaking/wagging with the predictions derived from the simulations of Tao et al. ${ }^{32-34}$ However, our experimental data of the oscillation periods at low shear rates significantly deviates from these prior simulations at lower shear rates, indicating some discrepancy between experiments and simulations of sheared nematic polymers. In summary, our results provide insight into the behavior of unique and coexisting sheared nematic director states, and how these states influence bulk rheological properties.

\section{Conflicts of interest}

There are no conflicts to declare.

\section{Acknowledgements}

R. J. F. acknowledges W.-R. Chen for access to the rheometer used in this work, and G.-R. Huang for helpful discussions regarding Fourier analysis. M. G. F. acknowledges support from the National Science Foundation under award number DMS-1517274, and discussions with Q. Wang and R. Zhou. R. J. F., M. G. F. and T. J. D. acknowledge support from a University of North Carolina at Chapel Hill Creativity Hubs award. This material is based upon work supported by the U.S. Department of Energy, Office of Science, Office of Workforce Development for Teachers and Scientists, Office of Science Graduate Student Research (SCGSR) program. The SCGSR program is administered by the Oak Ridge Institute for Science and Education (ORISE) for the DOE. ORISE is managed by ORAU under contract number DE-SC0014664. All opinions expressed in this paper are the authors' and do not necessarily reflect the policies and views of DOE, ORAU, or ORISE. This material is based upon work supported by, or in part by, the U.S. Army Research Laboratory and the U.S. Army Research Office under contract number W911NF-16-1-035. This work was performed in part at the Duke University Shared Materials Instrumentation Facility (SMIF), member of the North Carolina Research Triangle Nanotechnology Network (RTNN), which is supported by the National Science Foundation, Grant ECCS-1542015, as part of the National Nanotechnology Coordinated Infrastructure ( $\mathrm{NNCl).}$

\section{References}

1. S. Hess, Z. Naturforsch., 1976, 31 a, 1507-1513.

2. M. Doi, J. Polymer Sci. Phys., 1981, 19, 229-243.

3. M. G. Forest, Q. Wang and R. Zhou, Rheol. Acta, 2004, 43, 17-37.

4. M. G. Forest, Q. Wang and R. Zhou, Rheol. Acta, 2004, 44, 80-93.

5. V. M. Ugaz, W. R. Burghardt, W. Zhou and J. A. Kornfield, Journal of Rheology, 2001, 45, 1029-1063.

6. M. Mortier, P. Moldenaers and J. Mewis, Rheol Acta, 1996, 35, 57-68.

7. P. Moldenaers, M. Mortier and J. Mewis, Chem. Eng. Sci., 1994, 49, 699-707.

8. K. Hongladarom and W. R. Burghardt, Macromolecules, 1993, 26, 785-794.

9. S. J. Picken, J. Aerts, H. L. Doppert, A. J. Reuvers and M. G. Northolt, Macromolecules, 1991, 24, 1366-1375.

10. S. M. Guskey and H. H. Winter, Journal of Rheology, 1991, 35, 1191-1207.

11. G. Marrucci and P. L. Maffettone, J. Rheol., 1990, 34, 1217-1230.

12. N. Grizzuti, S. Cavella and P. Cicarelli, Journal of Rheology, 1990, 34, 1293-1310.

13. J. Mewis and P. Moldenaers, Mol. Cryst. Liq. Cryst., 1987, 153, 291-300.

14. G. G. Viola and D. G. Baird, Journal of Rheology, 1986, 30, 601-628.

$15 . \quad$ R. G. Larson, Macromolecules, 1990, 23, 3983-3992.

16. G. Marrucci and P. L. Maffettone, Macromolecules, 1989, 22, 4076-4082.

17. G. Marrucci and P. L. Maffettone, J. Rheol., 1990, 34, 1231-1244.

18. Y. Farhoudi and A. D. Rey, J. Rheol., 1993, 37, 289-314.

19. R. G. Larson and H. C. Ottinger, Macromolecules, 1991, 24, 6270-6282.

20. M. G. Forest and Q. Wang, Rheol. Acta, 2003, 42, 20-46.

21. S. Hess and M. Kröger, J. Phys. Condens. Matter, 2004, 16, S3835-S3859.

22. M. Grosso, S. Crescitelli, E. Somma, J. Vermant, P. Moldenaers and P. L. Maffettone, Phys. Rev. Lett., 2003, 90, 098304.

23. G. Rienacker, M. Kroger and S. Hess, Phys. Rev. E, 2002, 66, 040702.

24. G. Rienacker, M. Kroger and S. Hess, Physica A, 2002, 315, 537-568.

25. M. Grosso, R. Keunings, S. Crescitelli and P. L. Maffettone, Phys. Rev. Lett., 2001, 86, 3184-3187.

26. C. V. Chaubal and L. G. Leal, J. Polym. Sci.: Polym. Phys. Ed., 1999, 37, 281-300.

27. F. Greco, M. Minale and G. Marrucci, J. Non-Newtonian Fluid Mech., 1998, 76, 351-362.

28. F. Greco and G. Marrucci, Liquid Crystals, 1997, 22, 11-16.

29. J. Mewis, M. Mortier, J. Vermant and P. Moldenaers, Macromolecules, 1997, 30, 1323-1328.

30. M. P. Lettinga, Z. Dogic, H. Wang and J. Vermant, Langmuir, 2005, 21, 8048-8057.

31. M. P. Lettinga and J. K. G. Dhont, J. Phys: Condens. Matter, 2004, 16, S3929-S3939.

32. Y. G. Tao, W. K. den Otter and W. J. Briels, Europhys. Lett., 2009, 86. 
33. Y. G. Tao, W. K. den Otter and W. J. Briels, J. Chem. Phys., 2006, 124, 204902.

34. Y. G. Tao, W. K. den Otter and W. J. Briels, Phys. Rev. Lett., 2005, 95, 237802.

35. T. Tsuji and A. D. Rey, Journal of Non-Newtonian Fluid Mechanics, 1997, 73, 127-152.

36. A. D. Rey and M. M. Denn, Annu. Rev. Fluid Mech., 2002, 34, 233-266.

$37 . \quad J$. Gao, Y. Wang, B. Norder, S. J. Garcia, S. J. Picken, L. A. Madsen and T. J. Dingemans, J. Membr. Sci., 2015, 489, 194-203.

38. N. Sarkar and D. Kershner, J. Appl. Polym. Sci., 1996, 62, 393-408.

39.

R. J. Fox, D. Yu, M. Hegde, A. S. Kumbhar, L. A. Madsen and T. J. Dingemans, ACS Appl. Mater. Interfaces, 2019, 11, 40564-40574.

40

W. M. Kulicke, G. Kiss and R. S. Porter, Rheol. Acta, 1977, 16, 568-572.

41. J. Ilavsky, J. Appl. Cryst., 2012, 45, 324-328.

42. W. Yang, H. Furukawa, Y. Shigekura, K. Shikinaka, Y. Osada and J. P. Gong, Macromolecules, 2008, 41, 1791-1799.

43. Y. Wang, J. Gao, T. J. Dingemans and L. A. Madsen, Macromolecules, 2014, 47, 2984-2992.

44. Y. Wang, Y. Chen, J. Gao, H. G. Yoon, L. Jin, M. Forsyth, T. J. Dingemans and L. A. Madsen, Adv. Mater., 2016, 28, 2571-2578.

45. Y. Wang, Y. He, Z. Yu, J. Gao, S. Ten Brinck, C. Slebodnick, G. B. Fahs, C. J. Zanelotti, M. Hegde, R. B. Moore, B. Ensing, T. J. Dingemans, R. Qiao and L. A. Madsen, Nat Commun, 2019, 10, 801

46. M. Hegde, L. Yang, F. Vita, R. J. Fox, R. v. d. Watering, B. Norder, U. Lafont, O. Francescangeli, L. A. Madsen, S. J. Picken, E. T. Samulski and T. J. Dingemans, Nat. Commun., 2020, 11.

47. R. F. Kayser and H. J. Raveché, Phys. Rev. A, 1978, 17, 2067-2072.

48. G. J. Vroege and H. N. W. Lekkerkerker, Rep. Prog. Phys., 1992, 55, 1241-1309.

49. S. Viale, A. S. Best, E. Mendes, W. F. Jager and S. J. Picken, Chem. Commun., 2004, 14, 1596-1597.

50.

E. Mendes, S. Viale, O. Santin, M. Heinrich and S. J. Picken, J. Appl. Cryst., 2003, 36, 1000-1005.

51. J. E. Bostwick, C. J. Zanelotti, C. lacob, A. G. Korovich, L. A. Madsen and R. H. Colby, Macromolecules, 2020, 53, 14051414.

52. G. Marrucci, Pure \& Appl. Chem., 1985, 57, 1545-1552.

53. R. G. Larson, Rheol. Acta, 1996, 35, 150-159.

54. A. D. Rey, Molecular Crystals and Liquid Crystals Science and Technology. Section A. Molecular Crystals and Liquid Crystals, 1993, 225, 313-335.

55. N. J. Alderman and M. R. Mackley, Faraday Discuss. Chem Soc., 1985, 79, 149-160.

56. R. G. Larson and D. W. Mead, Liquid Crystals, 1992, 12 751-768.

57. S. Onogi and T. Asada, Rheology and Rheo-Optics of Polymer Liquid Crystals, 1980.

58. L. Walker and N. Wagner, J. Rheol., 1994, 38, 1525-1547.

59. L. M. Walker, W. A. Kernick and N. J. Wagner, Macromolecules, 1997, 30, 508-514.

$60 . \quad$ V. M. Ugaz, J. D. K. Cinader and W. R. Burghardt, Macromolecules, 1997, 30, 1527-1530.
61. L. M. Walker, M. Mortier and P. Moldenaers, Journal of Rheology, 1996, 40, 967-981.

62. L. M. Walker, N. J. Wagner, R. G. Larson, P. A. Mirau and P. Moldenaers, Journal of Rheology, 1995, 39, 925-952.

63. P. Moldenaers, G. Fuller and J. Mewis, Macromolecules, 1989, 22, 960-965.

64. W. R. Burghardt and G. G. Fuller, Macromolecules, 1991, 24.

65. R. G. Larson and M. Doi, Journal of Rheology, 1991, 35, 539-563.

66. R. G. Larson, Macromolecules, 1990, 23, 3983-3992.

67. G. Kiss and R. S. Porter, J. Polymer Sci. Phys., 1980, 18, 361-388.

68. G. Kiss and R. S. Porter, Journal of Polymer Science: Polymer Symposium, 1978, 65, 193-211.

69. M. Vasudevan, E. Buse, D. Lu, H. Krishna, R. Kalyanaraman, A. Q. Shen, B. Khomami and R. Sureshkumar, Nat Mater, 2010, 9, 436-441.

70. C.-H. Liu and D. J. Pine, Phys. Rev. Lett., 1996, 77, 2121 2124.

71. A. Perazzo, J. K. Nunes, S. Guido and H. A. Stone, Proc. Natl. Acad. Sci. U.S.A., 2017, 114, E8557-E8564.

72. S. Rendon, W. R. Burghardt and R. A. Bubeck, Rheologica Acta, 2007, 46, 945-956.

73. P. Pakdel and G. H. McKinley, Phys. Rev. Lett., 1996, 77, 2459-2462.

74. J. J. Magda and R. G. Larson, J. Non-Newtonian Fluid Mech., 1988, 30, 1-19.

$75 . \quad J$. J. Magda, S. G. Baek, K. L. DeVris and R. G. Larson, Polymer, 1991, 32, 1794-1796.

76. J. J. Magda, S.-G. Baek, K. L. DeVries and R. G. Larson, Macromolecules, 1991, 24, 4460-4468.

77. S. G. Baek, J. J. Magda, R. G. Larson and S. D. Hudson, J. Rheol., 1994, 38, 1473-1503.

78. M. Doi and S. F. Edwards, The Theory of Polymer Dynamics, Oxford University Press, 1986.

79. K. R. Purdy, Z. Dogic, S. Fraden, A. Ruhm, L. Lurio and S. G. Mochrie, Phys Rev E Stat Nonlin Soft Matter Phys, 2003, 67, 031708.

80. V. Faraoni, M. Grosso, S. Crescitelli and P. L. Maffettone, J. Rheol., 1999, 43, 829-843.

81. C. Lang, J. Kohlbrecher, L. Porcar, A. Radulescu, K. Sellinghoff, J. K. G. Dhont and M. P. Lettinga, Macromolecules, 2019, 52, 9604-9612.

82. F. Cocchini, M. R. Nobile and D. Acierno, J. Rheol., 1992, 36, 1307-1311.

83. S. G. Baek, J. J. Magda and R. G. Larson, J. Rheol., 1993, 37, 1201-1224.

84. S. G. Baek, J. J. Magda and S. Cementwala, J. Rheol., 1993, 37, 935-945.

85. W.-J. Zhou, J. A. Kornfield, V. M. Ugaz, W. R. Burghardt, D. R. Link and N. A. Clark, Macromolecules, 1999, 32, 55815593.

86. F. Cocchini, M. R. Nobile and D. Acierno, J. Rheol., 1991, 35, 1171-1189.

87. A. Romo-Uribe and A. H. Windle, Macromolecules, 1993, 36, 7100-7102.

88. E. O. Brigham, The Fast Fourier Transform and Its Applications, Prentice-Hall, Inc, 1988.

89. F. E. Caputo and W. R. Burghardt, Macromolecules, 2001 34, 6684-6694. 
90. F. E. Caputo, V. M. Ugaz, W. R. Burghardt and J.-F. Berret, J. Rheol., 2002, 46. 